\title{
Risk Factors of Hyponatremia in Children with Lower Respiratory Tract Infection (LRTI)
}

\author{
Chinmaya Mahapatra1, (1) Vinod Kumar Sharma2, (1) Siddhant Singhal2, (1) Roshan Kumar Jangid2, \\ (1) Tagaram Karthik Laxminath2
}

${ }^{1}$ All India institute of Medical Sciences, Department of Pediatrics and Neonatology, Bhubaneswar, India

2Den Dayal Upadhyay Hospital, Clinic of Pediatrics, New Delhi, India

\begin{abstract}
Aim: Lower respiratory tract infection (LRTI) is a serious illness especially in children under 5 years of age. Hyponatremia is the most common electrolyte abnormality seen in hospitalized children. This study aimed to evaluate the correlation between hyponatremia in children admitted to a paediatric ward, in the setting of acute LRTI in different age groups and to determine the association of hyponatremia with different types of acute LRTI in children.

Materials and Methods: This study included 231 clinically diagnosed children (1 month to 12 years) with acute LRTI. General examinations and systemic examinations were performed. Laboratory investigations included serum electrolyte level ( $\mathrm{Na}+$ ) and radiological investigations included chest X-ray. The conditions observed in the children included bronchopneumonia (BPN), lobar pneumonia, wheeze-associated LRTI (WALRI), bronchiolitis, and empyema. Statistical analysis was carried out using SPSS 16.0 version. A p-value $<0.05$ was considered statistically significant.

Results: Out of 231 cases, hyponatremia was present in 136 cases (58.9\%). Mild, moderate, and severe hyponatremia were present in $83.8 \%$, $13.2 \%$, and $2.9 \%$ patients, respectively. Most of the patients with BPN had mild hyponatremia (89\%). The prevalence of hyponatremia was significantly higher in children aged 6-10 years [odds ratio $(O D)=4.29,95 \%$ confidence interval $(C I)=0.90-20.45, p<0.05$ ], females $(O R=0.56$, $95 \% \mathrm{Cl}=0.32-0.96, \mathrm{p}=0.03)$ and cases of empyema ( $\mathrm{OR}=4.49,95 \% \mathrm{Cl}=1.48-13.60, \mathrm{p}=0.008)$.

Conclusion: In conclusion, among children hospitalized with LRTI, an older age (6-10 years), being female, and the presence of empyema are significant risk factors for the development of hyponatremia. In such children, serum electrolytes should be regularly measured to prevent adverse clinical outcomes.
\end{abstract}

Keywords: Bronchopneumonia, electrolyte, hyponatremia, lower respiratory tract infection

\section{Introduction}

Lower respiratory tract infection (LRTI) is an inflammation of the airways (pulmonary tissue), due to viral or bacterial infection, below the level of the larynx. LRTI includes various diseases such as: bronchiolitis, wheezeassociated LRTI (WALRI), bronchopneumonia (BPN), Lobar pneumonia and Empyema. LRTI is one of the serious illnesses requiring hospitalization especially in children under 5 years of age. It accounts for $30 \%$ of deaths annually worldwide mostly due to pneumonia as the leading cause (1).

In the last 10 years, changes have been observed in the epidemiology of LRTIs as there has been a reduction in the burden in children below 5 years of age and an increased burden has been observed in patients over 70 years of age. 
Among all ages, pneumococcal pneumonia was responsible for $55.4 \%$ of LRTI deaths (2).

Patients with pneumonia and bronchiolitis, the most common diseases encountered in paediatric general practice, are at particular risk of developing hyponatremia due to anti-diuretic hormone (ADH) over-secretion (3-5).

Several ongoing studies are being conducted to determine any correlation between hyponatremia and acute LRTI in children as there is growing interest in the issue of sodium imbalance in different types of LRTI (1). Singh et al. (6) conducted a study on hyponatremia in different types of LRTI and found hyponatremia in 33\% cases out of a total 100 cases (51.5\% mild, 42.5\% moderate and 6\% severe). A similar study conducted by Chaitra et al. (1) found hyponatremia in $45 \%$ out of 91 cases $(70.7 \%$ mild, $21.9 \%$ moderate and $7.3 \%$ severe). However, there are few studies on Indian children and the western data may not aptly apply to the Indian subpopulation.

This study was carried out to characterize the association between hyponatremia in those patients admitted to a paediatric ward, in the setting of acute LRTI in different age groups and to determine the association between the severity of hyponatremia and different types of acute LRTI in children.

\section{Methodology}

A prospective observational study was conducted on the paediatric age group ( 1 month to 12 years) presenting with symptoms and signs suggestive of acute LRTI from December 2017 to June 2019. This study was approved by the Institutional Ethical Committee. After the child had met the inclusion criteria, informed consent was obtained from the guardian or the parent after explaining the aims of the study to him or her.

\section{Exclusion criteria:}

- Known case of congenital heart disease

- Known case of renal disease

- History of recent surgery

- Any child with a previous diagnosis of syndrome of inappropriate antidiuretic hormone

- Known case of bronchial asthma

- Febrile seizures

- Patient with acute gastroenteritis

- Cystic fibrosis

- Pan-hypopituitarism

- Hypothyroidism
- Metabolic diseases

- Chromosomal or genetic disorders

- Meningitis

The sample size calculation was based on the prevalence of hyponatremia as seen in the study of Chaitra et al. (1) among LRTI children. A sample size of 170 children was calculated by means of Fisher's formula.

Complete history, physical examination, relevant laboratory investigation, and radiological investigations were examined to confirm the diagnosis. Pneumonia was diagnosed in patients presenting with fever, tachypnoea, dyspnoea, grunting, and chest retraction. BPN and lobar pneumonia were further differentiated on the basis of chest X-ray (patchy opacity in BPN and lobar involvement in lobar pneumonia). WALRI was diagnosed as signs and symptoms of respiratory obstruction and wheeze without any family history or atopy. Wheezing refers to a highpitched whistling sound audible without auscultation. Bronchiolitis was confirmed on the basis of tachypnoea, rhinorrhoea, crepitation, wheeze, subcostal and intercostal retraction, nasal flaring, grunting and chest $X$-ray showing hyperinflation. Empyema was characterized by systemic toxicity, breathing difficulty, decreased movement of the chest, decreased air entry, dull percussion note, and obliteration of the costophrenic angle with varying degrees of opacification in chest X-ray.

After obtaining consent from the parent, $2 \mathrm{~mL}$ of blood was taken from each patient using the aseptic technique and this sample was put in a vacutainer. The blood samples were then transported to the emergency laboratory within an hour of collection for analysis and reports were collected. Analysis was carried out to determine for which particular group of respiratory illness was the association of hyponatremia more significant. The patients were divided into age groups as 1 month to 12 months, $1-3$ years (12 months to 36 months), 3-6 years, 6-10 years, and $10-12$ years. Serum sodium level was measured in our hospital based on the principle of indirect ion selective electrode with the Beckman Coulter AU480 fully automated biochemistry analyser.

The prevalence of hyponatremia and the type of LRTI was noted in the children. Hyponatremia was graded into mild, moderate and severe grades.

\section{Statistical Analysis}

The results are presented as frequencies, percentages and mean \pm standard deviation. The chi-square test was used to assess the associations. The unpaired t-test was used 
to compare continuous variables. Binary logistic regression analysis was carried out to determine the strength of association. The odds ratio (OR) with its $95 \%$ confidence interval $(\mathrm{Cl})$ was calculated. A p-value $<0.05$ was considered significant. All the analysis was carried out on SPSS 16.0 version (Chicago, Inc. USA).

\section{Results}

The mean age of the study patients was $1.88 \pm 2.41$ years with 146 (63.2\%) males and 85 (36.8\%) females.

Out of all the cases of LRTI, there was the highest number of cases for BPN (61\%), followed by empyema (12.6\%), lobar pneumonia (9.5\%), bronchiolitis (9.1\%) and the lowest number of cases of WALRI (7.8\%) (Figure 1).

The prevalence of hyponatremia was found to be $58.9 \%$ (Out of 231 cases, hyponatremia was present in 136 cases and absent in 95 cases). Mild hyponatremia was the most common $(83.8 \%)$ followed by moderate $(13.2 \%)$ and then by severe hyponatremia (2.9\%).

About half of the patients were 1-12 months (51.5\%) followed by $1-3$ years (35.1\%), 3-6 (6.9\%), 6-10 years $(5.2 \%)$ and lastly $10-12$ years $(1.3 \%)$.

The prevalence of hyponatremia was highest in the age group 10-12 years (100\%) and lowest in the age group 1-12 months (53.8\%). However, as the number of cases was only three in the age group 10-12 years, we cannot make any conclusion on this basis. Prevalences in the 6-10 years group, the 1-3 years group and the 3-6 years group were $83.3 \%, 61.7 \%$ and $56.2 \%$ respectively. The prevalence of hyponatremia was 4.29 times higher in the 6-10 years group than the 1-12 months group $(\mathrm{OR}=4.29,95 \% \mathrm{Cl}=0.90-20.45$, p<0.05) (Table I).

The prevalence of hyponatremia was higher in females $(66.7 \%)$ than males (53\%) with a significantly higher risk (OR=0.56, 95\% Cl=0.32-0.96, p=0.03) (Table II).

The prevalence of hyponatremia was highest in empyema (86.2\%) and was lowest in WALRI (22.2\%). The risk association showed a significantly increased risk of hyponatremia in empyema $(\mathrm{OR}=4.49,95 \% \mathrm{Cl}=1.48-13.60$, $\mathrm{p}=0.008)$ and decreased risk in WALRI (OR $=0.20,95 \%$ $\mathrm{Cl}=0.06-0.65, \mathrm{p}=0.008$ ) (Table III).

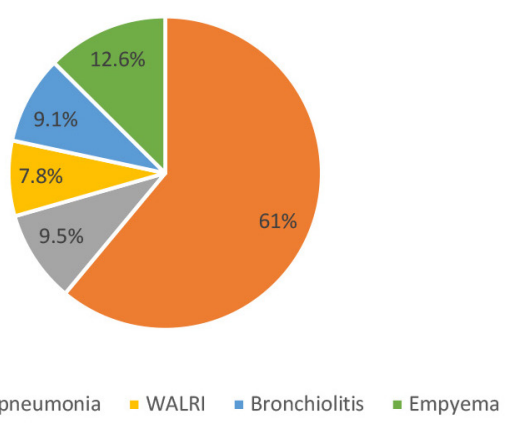

Figure 1. Shows the overall distribution of LRTI

BPN: Bronchopneumonia, LRTI: Lower respiratory tract infection, WALRI: Wheeze-associated LRTI

\begin{tabular}{|c|c|c|c|c|c|c|c|c|}
\hline \multirow{3}{*}{ Age in months } & \multirow{3}{*}{$\begin{array}{l}\text { No. of patients } \\
\text { No. }\end{array}$} & & \multicolumn{4}{|c|}{ Hyponatremia } & \multirow{3}{*}{ OR $(95 \% \mathrm{Cl})$} & \multirow{3}{*}{ p-value } \\
\hline & & \multicolumn{2}{|c|}{ Present } & \multicolumn{2}{|c|}{ Absent } & \multirow[b]{2}{*}{$\%$} & & \\
\hline & & $\%$ & No. & $\%$ & No. & & & \\
\hline 1-12 months & 119 & 51.5 & 64 & 53.8 & 55 & 46.2 & 1.00 (Ref.) & \\
\hline $1-3$ years & 81 & 35.1 & 50 & 61.7 & 31 & 38.3 & $1.38(0.78-2.46)$ & 0.26 \\
\hline 3-6 years & 16 & 6.9 & 9 & 56.2 & 7 & 43.8 & $1.10(0.38-3.16)$ & 0.85 \\
\hline $6-10$ years & 12 & 5.2 & 10 & 83.3 & 2 & 16.7 & $4.29(0.90-20.45)$ & 0.06 \\
\hline $10-12$ years & 3 & 1.3 & 3 & 100.0 & 0 & 0.0 & - & - \\
\hline
\end{tabular}

Table II. Comparison of prevalence of hyponatremia with gender in LRTI

\begin{tabular}{|c|c|c|c|c|c|c|c|c|}
\hline \multirow{3}{*}{ Gender } & \multirow{3}{*}{\begin{tabular}{|l} 
No. of patients \\
No.
\end{tabular}} & & \multicolumn{4}{|c|}{ Hyponatremia } & \multirow{3}{*}{ OR $(95 \% \mathrm{Cl})$} & \multirow{3}{*}{ p-value } \\
\hline & & \multicolumn{2}{|c|}{ Present } & \multicolumn{2}{|c|}{ Absent } & \multirow[b]{2}{*}{$\%$} & & \\
\hline & & $\%$ & No. & $\%$ & No. & & & \\
\hline Male & 132 & 57.1 & 70 & 53.0 & 62 & 47.0 & $0.56(0.32-0.96)$ & $0.03^{*}$ \\
\hline Female & 99 & 42.9 & 66 & 66.7 & 33 & 33.3 & 1.00 (Ref.) & \\
\hline
\end{tabular}


Based on the type of LRTI (Table III):

1) BPN had $89 \%$ mild hyponatremia, $9.8 \%$ moderate hyponatremia and $1.2 \%$ severe hyponatremia.

2) Lobar pneumonia had $85.7 \%$ mild hyponatremia, $14.3 \%$ moderate hyponatremia and 0\% severe hyponatremia.

3) WALRI had 100\% mild hyponatremia.

4) Bronchiolitis had $72.7 \%$ mild hyponatremia, $27.3 \%$ moderate hyponatremia and 0\% severe hyponatremia.

5) Empyema had 68\% mild hyponatremia, 20\% moderate hyponatremia and $12 \%$ severe hyponatremia.

\section{Discussion}

Hyponatremia is a significant electrolyte abnormality commonly seen in hospital-admitted children with LRTI. The underlying mechanism is the release of excess ADH (7-10). The current study has importance as it illustrates an association of hyponatremia with different types of LRTI among children $(11,12)$.

Literature reports hyponatremia to be in the range of $6 \%$ to $73 \%$. Our results fell within the reported range as we found hyponatremia in 136 (58.9\%) cases.

Out of all the cases of hyponatremia, $83.8 \%$ cases had mild hyponatremia, and $13.2 \%$ and $2.9 \%$ cases had moderate and severe hyponatremia, respectively. Our findings corroborated the previous studies which reported the majority of cases with mild hyponatremia (13-18). Among these studies, the prevalence of mild cases of hyponatremia was significantly more in developed countries when compared to developing countries; thus highlighting the improved care and management in developed countries.

Our study results showed that the age group of 6-10 years carried a significantly increased risk of hyponatremia in relation to other age groups $(\mathrm{OR}=4.29,95 \% \mathrm{Cl}=0.90-20.45$, $p>0.05)$. This may be because the inherent capacity of the body to balance the electrolytes decreases with advancing age in children. Secondly, it could be because children of less than 5 years of age are managed more effectively and care is given to prevent the occurrence of hyponatremia. Among the previous studies, the association of hyponatremia has been studied mainly in the age group of 2-month-old to 5 -year-old children (18) and thus very few studies have reported on the age association of hyponatremia. Wrotek and Jackowska (19) found that the age group of $>4$ years has a greater chance of hyponatremia and severe infection. Park et al. (13) found that increasing age is an independent risk factor for the development of hyponatremia (OR=1.007, 95\% Cl 1.002-1.012, p=0.006). Our study has strength in validating this relationship of hyponatremia with age as this may warn clinicians who manage children.

Another interesting association seen in the present study was that females carried a significantly increased risk of hyponatremia when compared to males. Relatively few studies have reported on the demographic association of children with hyponatremia and some with contrasting results to the present study. Park et al. (13) found that males are more prone to hyponatremia (OR=1.361, 95\% Cl 1.1051.675, $p=0.004$ ) and Sakellaropoulou et al. (20) found no significant difference in the gender distribution of children having hyponatremia. The higher risk of hyponatremia in females in the present study may be due to their late presentation in the hospital since ours is a male dominant society where medical care for female children may not be availed at an early stage.

In our endeavour to determine the relationship of hyponatremia with different etiologies of LRTI, it was observed that hyponatremia carried a significantly higher risk in empyema (OR=4.49,95\% $\mathrm{Cl}=1.48-13.60, \mathrm{p}=0.008)$ and lower risk in WALRI $(\mathrm{OR}=0.20,95 \% \mathrm{Cl}=0.06-0.65$, $p=0.008)$. This may be ascribed to the seriousness of the disease. On one hand, empyema is a bacterial disease serious enough to require invasive treatment modalities

Table III. Comparison of prevalence of hyponatremia with type of LRTI

\begin{tabular}{|c|c|c|c|c|c|c|c|c|}
\hline \multirow{3}{*}{ Type of LRTI } & \multirow{3}{*}{$\begin{array}{l}\text { No. of patients } \\
\text { No. }\end{array}$} & & \multicolumn{4}{|c|}{ Hyponatremia } & \multirow{3}{*}{ OR $(95 \% \mathrm{Cl})$} & \multirow{3}{*}{ p-value } \\
\hline & & \multicolumn{2}{|c|}{ Present } & \multicolumn{2}{|c|}{ Absent } & \multirow[b]{2}{*}{$\%$} & & \\
\hline & & $\%$ & No. & $\%$ & No. & & & \\
\hline BPN & 141 & 61.0 & 82 & 58.2 & 59 & 41.8 & 1.00 (Ref.) & \\
\hline Lobar pneumonia & 22 & 9.5 & 14 & 63.6 & 8 & 36.4 & $1.25(0.49-3.19)$ & 0.62 \\
\hline WALRI & 18 & 7.8 & 4 & 22.2 & 14 & 77.8 & $0.20(0.06-0.65)$ & $0.008^{*}$ \\
\hline Bronchiolitis & 21 & 9.1 & 11 & 52.4 & 10 & 47.6 & $0.79(0.31-1.98)$ & 0.61 \\
\hline Empyema & 29 & 12.6 & 25 & 86.2 & 4 & 13.8 & $4.49(1.48-13.60)$ & $0.008^{*}$ \\
\hline
\end{tabular}


of pus drainage and culture sensitivity; and on the other hand, WALRI is a viral infection causing wheezing in the chest which can be managed well. This also corroborated with the findings wherein mild cases of hyponatremia were observed in all children with WALRI, whereas in empyema, $32 \%$ had moderate to severe hyponatremia. Other clinical conditions such as BPN, lobar pneumonia and bronchiolitis were intermediate in the risk for hyponatremia without any statistical significance $(p>0.05)$. In the study by Park et al. (13), children with acute tonsillopharyngitis had the highest incidence of hyponatremia (44.1\%). Kaneko (21) found that hyponatremia was more common when the deeper inflammatory sites of the respiratory tract were involved such as in cases of pharyngitis (13.3\%), bronchitis or bronchiolitis (22.9\%), and pneumonia (38.7\%).

One factor causing increased hyponatremia in empyema in our study could be because such children have high TLC counts and inflammation. Although the present study did not assess WBC counts or CRP levels, Park et al. (13) found a significantly higher WBC counts and CRP levels in acute tonsillopharyngitis, indicating that increasing inflammation may be associated with the severity of hyponatremia.

The underlying mechanism remains an enigma although current concepts favour the involvement of syndrome of inappropriate secretion of antidiuretic hormone (SIADH) in the causation of hyponatremia. It has been proposed that fever resets the hypothalamic thermostat and osmostat (for ADH secretion) in children. The new osmostat increases the secretion of $A D H$, causing fluid retention and decreased osmolarity in the body, thereby leading to hyponatremia $(8,10)$.

Based on the current study results and the study by Park et al. (13), it can be postulated that SIADH may be the result of inflammation wherein the cytokines surge of interleukin (IL)-1 $\beta$ and IL- 6 form the primary mediators causing increased ADH secretion and SIADH. This has been indirectly evidenced in a study on rats which showed that IL-1 $\beta$ stimulated both the peripheral and central release of vasopressin (22). Further, the stimulated release of arginine vasopressin (AVP) by IL-6 was seen in the study by Palin et al. (23) through "magnocellular AVP-secreting neurons". Previously, two studies on pneumonia observed an inverse relationship between inflammatory markers and serum sodium levels $(19,24)$. In our cohort, we also found that hyponatremia was significantly increased in cases of empyema among various LRTI, and the risk association was significant with an OR of 4.49 .
Literature is sparse regarding studies which evaluated the factors affecting the fall in sodium levels in children with respiratory tract infections (13). Our study has strength in showing an age and gender association with hyponatremia in children with LRTI. Additionally, we also determined the etiological risk associated with hyponatremia. However, we failed to determine the role of co-infection in hyponatremia.

\section{Study Limitations}

Our study was also limited by its cross-sectional design wherein we could not suggest the association of hyponatremia with hospitalisation and mortality. Another limitation of the study was that it was conducted in general paediatric ward, and not in an ICU. This might have created a potential bias in the prevalence of hyponatremia among different children with LRTI. Lastly, since our study centre was a referral centre, many sick babies were referred to the hospital from various hospitals and their treatment status was not known.

\section{Conclusion}

In conclusion, among those children hospitalized with LRTI, an older age (6-10 years), being female, and the presence of empyema are significant risk factors for the development of hyponatremia. In such children, serum electrolytes should be regularly measured to prevent adverse clinical outcomes.

\section{Ethics}

Ethics Committee Approval: The study was approved from the Institutional Review Board Ethical Committee (IEC/ROHTAK/2018/16-4).

Informed Consent: Informed consent was obtained from the guardian or the parent after explaining the aims of the study to him or her.

Peer-review: Externally peer-reviewed.

\section{Authorship Contributions}

Design: S.S., R.K., R.K.J., Data Collection or Processing: S.S., R.K., R.K.J., T.K.L., Analysis or Interpretation: C.M., R.K.J., Literature Search: S.S., C.M., R.K., R.K.J., Writing: R.B.G.B., R.D.G.

Conflict of Interest: No conflict of interest was declared by the authors.

Financial Disclosure: The authors declared that this study received no financial support. 


\section{References}

1. Chaitra KM, Kumar MN, Reddy SC. Hyponatremia in lower respiratory tract infections. Int / Contemp Pediatr 2016; 3:381-4.

2. Feldman C, Shaddock E. Epidemiology of lower respiratory tract infections in adults. Expert Rev Respir Med 2019; 13:63-77.

3. Szabo FK, Lomenick JP. Syndrome of inappropriate antidiuretic hormone secretion in an infant with respiratory syncytial virus bronchiolitis. Clin Pediatr (Phila) 2008; 47:840-2.

4. Van Steensel-Moll HA, Hazelzet JA, van der Voort E, Neijens HJ, Hackeng WH. Excessive secretion of antidiuretic hormone in infections with respiratory syncytial virus. Arch Dis Childhood 1990; 65:1237-9.

5. Hanna S, Tibby SM, Durward A, Murdoch IA. Incidence of hyponatraemia and hyponatraemic seizures in severe respiratory syncytial virus bronchiolitis. Acta Paediatr 2003; 92:430-4.

6. Singh $\mathrm{P}$, Vandana, Attri $\mathrm{HK}$, et al. A study of hyponatremia in lower respiratory infections in children aged 2 months to 5 years. Ann Int Med Den Res 2019;5(2):PE16-20.

7. Duru NS, Civilibal M, Bozdogan S, Elevli M. Hyponatremia in children hospitalized with pneumonia. / Pediatr Infect 2013; 7:102.

8. Kanai H, Sato $Y$, Ichihashi K. Hyponatremia in patients with respiratory syncytial virus bronchiolitis. Pediatr Health Med Ther 2012; 3:39-43.

9. Seifert ME, Welak SR, Carroll CL. Hyponatremia is associated with increased severity of disease in critically ill children with bronchiolitis. Int J Clin Med 2010; 1:37-40.

10. Rahul V, Jose O. Prevalence of hyponatremia in children with pneumonia-cross-sectional study. IOSR / Dent Med Sci 2017; 16:46-50.

11. Skippen $\mathrm{P}$, Adderley $\mathrm{R}$, Bennett $\mathrm{M}$, et al. latrogenic hyponatremia in hospitalized children: Can it be avoided? Paediatr Child Health 2008; 13:502-6.

12. Mhaske S, Bulsara L, Kadam V. Correlation of hyponatremia in children presenting with acute lower respiratory infection in a tertiary care hospital tract. Antiseptic 2016; 113:24-7.
13. Park SW, Shin SM, Jeong M, et al. Hyponatremia in children with respiratory infections: a cross-sectional analysis of a cohort of 3938 patients. Sci Rep 2018; 8:16494.

14. Hasegawa K, Stevenson MD, Mansbach JM, et al. Association between hyponatremia and higher bronchiolitis severity among children in the ICU with bronchiolitis. Hosp Pediatr 2015; 5:3859.

15. Luu R, DeWittPE, Reiter PD, Dobyns EL, Kaufman J. Hyponatremia in children with bronchiolitis admitted to the pediatric intensive care unit is associated with worse outcomes. I Pediatr 2013; 163:1652-6.

16. Mandal PP, Garg M, Choudhary IP. To study the association and significance of hyponatremia in pneumonia in paediatric patients treated in hospital setting. Int I Contemp Med Res 2018:5:11-4.

17. Yilmaz $Y$, Candar $T$, Kara $F$, et al. Serum sodium levels in children with lung infections. I Pediatr Inf 2016; 10:10-3.

18. Praneetha CK, Ahirrao VS, Srinivasa K, Premalatha R. Hyponatremia in children of 2 months to 5 years of age with community acquired pneumonia and its correlation with severity of illness and outcome. Pediatr Rev 2019; 6:561-6.

19. Wrotek A, Jackowska T. Hyponatremia in children hospitalized due to pneumonia. Adv Exp Med Biol 2013; 788:103-8.

20. Sakellaropoulou A, Hatzistilianou $M$, Eboriadou $M$, Athanasiadou-Piperopoulou F. Hyponatraemia in cases of children with pneumonia. Arch Med Sci 2010; 6:578-83.

21. Kaneko KK. Hyponatremia in children with respiratory tract infection. Pediatr Nephrol 2009;24:1595; author reply 1597-8.

22. Landgraf R, Neumann I, Holsboer F, Pittman Q). Interleukin-1 beta stimulates both central and peripheral release of vasopressin and oxytocin in the rat. Eur / Neurosci 1995; 7:592-8.

23. Palin K, Moreau ML, Sauvant J, et al. Interleukin-6 activates arginine vasopressin neurons in the supraoptic nucleus during immune challenge in rats. Am J Physiol Endocrinol Metab 2009; 296:E1289-99. doi: 10.1152/ajpendo.90489.2008.

24. Don M, Valerio G, Korppi M, Canciani M. Hyponatremia in pediatric community-acquired pneumonia. Pediatr Nephrol 2008; 23:2247-53. 